Pacific Journal of Mathematics

A TOPOLOGICAL CHARACTERIZATION OF COMPLETE, 


\title{
A TOPOLOGICAL CHARACTERIZATION OF COMPLETE, DISCRETELY VALUED FIELDS
}

\section{SETH WARNER}

\begin{abstract}
It is shown that the topology of a topological field $F$ is given by a complete, discrete valuation if and only if $F$ is locally strictly linearly compact. More generally, the topology of a topological division ring $K$ is given by a complete, discrete valuation and $K$ is finite dimensional over its center if and only if $K$ is locally centrally linearly compact, that is, if and only if $K$ contains an open subring $B$, the open left ideals of which form a fundamental system of neighborhoods of zero, such that $B$, regarded as a module over its center, is strictly linearly compact.
\end{abstract}

In [5], Jacobson showed that the topology of an indiscrete, totally disconnected, locally compact division ring is given by a discrete valuation (that is, a valuation whose value group is isomorphic to the cyclic group of integers). Consequently, an indiscrete topological division ring $K$ is locally compact and totally disconnected if and only if its topology is given by a complete, discrete valuation whose residue field is finite [4, Prop. 2, p. 118, Prop. 1, p. 156]. From this, it follows rather readily that the center $C$ of $K$ is indiscrete, that $K$ is finite dimensional over $C$, and that $C$ is either a finite extension of the $p$-adic number field for some prime $p$ or the field of formal power series over a finite field [4, Theorem 1, p. 158].

Our purpose here is to generalize Jacobson's theorem by characterizing those topological fields whose topology is given by a complete, discrete valuation, and more generally, those topological division rings $K$ such that $K$ is finite dimensional over its center and the topology of $K$ is given by a complete, discrete valuation.

For this purpose, we assume some familiarity with basic properties of linearly compact and strictly linearly compact modules and rings, as developed in [10] or [3, Exercises 14-22, pp. 108-112]. We recall that a (left) topological $A$-module $E$ (it is not assumed that $E$ is unitary) is linearly topologized if the open submodules of $E$ form a fundamental system of neighborhoods of zero; $E$ is linearly compact if $E$ is Hausdorff, linearly topologized, and every filter base of cosets of submodules has an adherent point; $E$ is strictly linearly compact if $E$ is linearly compact and every continuous epimorphism from $E$ onto a Hausdorff, linearly topologized $A$-module is open (equivalently, if $E / U$ is an artinian $A$-module for every open submodule $U$ of $E$ ). A topological ring $A$ is respectively linearly topologized, linearly compact, 
or strictly linearly compact if the associated left $A$-module $A$ is.

Definition. A topological ring $A$ is locally strictly linearly compact if $A$ has an open subring $B$ that is strictly linearly compact for its induced topology.

To handle the noncommutative case, we need the following definition:

Definition. A topological ring $B$ is centrally linearly compact if the open left ideals of $B$ form a fundamental system of neighborhoods of zero and if $B$, regarded as a module over its center $C_{B}$, is a strictly linearly compact $C_{B}$-module. A topological ring $A$ is locally centrally linearly compact if $A$ contains an open subring that is centrally linearly compact for its induced topology.

Thus a commutative topological ring is (locally) centrally linearly compact if and only if it is (locally) strictly linearly compact. Note that if $B$ is a centrally linearly compact ring, then for any subring $B_{0}$ of $B$ that contains the center $C_{B}, B$ is a strictly linearly compact $B_{0}$-module (in particular, $B$ is a strictly linearly compact ring); indeed, since the open left ideals of $B$ form a fundamental system of neighborhoods of zero, $B$ is a linearly topologized $B_{0}$-module, and since a $B_{0}$-submodule is also a $C_{B}$-submodule, every filter base of cosets of $B_{0}$-submodules necessarily has an adherent point.

By a topological division ring (field) $K$ we mean a topological ring that is algebraically a division ring (field); that is, we do not assume that $x \mapsto x^{-1}$ is continuous on the set $K^{*}$ of nonzero elements.

LEMMA 1. If $B$ is an open, centrally linearly compact subring of an indiscrete topological division ring $K$, then there is an open, centrally linearly compact subring $B_{1}$ of $K$ that contains 1 .

Proof. Let $B_{1}$ be the closure of the subring generated by $B$ and 1. The open left ideals of $B$ then form a fundamental system of neighborhoods of zero in $B_{1}$; each open left ideal $a$ of $B$ is a left ideal of $B_{1}$, for as $a$ is closed, $\left\{x \in B_{1}: x a \subseteq \mathfrak{a}\right\}$ is a closed subring of $B_{1}$ containing $B$ and 1 and hence is all of $B_{1}$.

Since $B$ is open, $B \neq(0)$; let $b$ be some nonzero element of $B$, and let $c$ be its inverse in $K$. Then, $B_{1}=B_{1} b c \leqq B_{1} B c$, so $B_{1} \subseteq B c$ since, as we saw above, $B$ is a left ideal of $B_{1}$. Thus $B c \supseteqq B_{1} \supseteqq B$, so $B c$ is a linearly topologized $C_{B}$-module, where $C_{B}$ is the center of $B$. Hence $B c$ is a strictly linearly compact $C_{B}$-module, as it is the image of the strictly linearly compact $C_{B}$-module $B$ under the continuous homomorphism $x \mapsto x c$. Consequently, the closed $C_{B}$-submodule 
$B_{1}$ of $B c$ is strictly compact; as $C_{B}$ is contained in the center of $B_{1}, B_{1}$ is a fortiori strictly linearly compact over its center.

We recall that an element $a$ of a topological ring is topologically nilpotent if $\lim a^{n}=0$.

Lemma 2. Let $K$ be a Hausdorff topological division ring, let $B$ be an open subring of $K$ that contains 1 , and let $\mathfrak{x}$ be the radical of $B$. If $B$ is strictly linearly compact, then $B$ is a (left) noetherian ring, $B / \mathfrak{r}$ is a division ring, the topology of $B$ is the $\mathfrak{r}$-adic topology, and $\mathfrak{x}$ is the set of all topological nilpotents of $B$.

Proof. As $B$ is open and as $y \mapsto y x$ is a homeomorphism for each $x \in K^{*}, B x$ is open for every $x \in K^{*}$, and hence every nonzero left ideal of $B$ is open. Let $\mathfrak{B}=\bigcap_{n=1}^{\infty} \mathfrak{x}^{n}$. Assume that $\mathfrak{B} \neq(0)$. Then $\mathfrak{B}$ is open, so $B / \mathfrak{s}$ is an artinian $B$-module and hence an artinian ring. Consequently, its radical $\mathfrak{r} / \mathfrak{s}$ is nilpotent, so there exists $n$ such that $\mathfrak{x}^{n}=\mathfrak{g}$. Hence $(0) \neq \mathfrak{x}^{n}=\mathfrak{x}^{n+1}=\cdots$, in contradiction to [10, Theorem 9]. Therefore, $\bigcap_{n=1}^{\infty} \mathfrak{x}^{n}=(0)$.

Since every nonzero left ideal of $B$ is open and hence closed, $B$ is a (left) noetherian ring, $B / \mathfrak{r}$ is an artinian ring, and the topology of $B$ is its $r$-adic topology by [13, Theorem 16]. Consequently, every element of $\mathfrak{r}$ is a topological nilpotent. Therefore, as $B$ is complete, $B$ is suitable for building idempotents [11, Lemma $4 ; 6$, Definition 1 , p. 53]. Thus every idempotent of $B / \mathfrak{x}$ is the coset of $\mathfrak{x}$ determined by an idempotent of $B$ [6, Proposition 4, p. 54]. But as $K$ is a division ring, $B$ has no idempotents other than 0 and 1 . Thus $B / \mathfrak{x}$ is an artinian, semisimple ring whose only idempotents are 0 and $1 . \mathrm{By}$ the Wedderburn-Artin theorem, therefore, $B / \mathfrak{r}$ is a division ring. In particular, if $x \notin \mathfrak{r}$, then $x+\mathfrak{r}$ is not a nilpotent of $B / \mathfrak{x}$, so $x$ is not a topological nilpotent.

THEOREM 1. If $K$ is an indiscrete, Hausdorff topological field, then the topology of $K$ is given by a complete, discrete valuation if and only if $K$ is locally strictly linearly compact.

Proof. Necessity. It is well known that a complete, semilocal noetherian ring, equipped with its natural $\mathfrak{x}$-adic topology, is strictly linearly compact [cf. 13, Corollary of Lemma 2]. In particular, the valuation ring of a complete discrete valuation is strictly linearly compact.

Sufficiency. By Lemma 1, there is an open, strictly linearly compact subring $B$ of $K$ that contains 1 . By Lemma $2, B$ is a complete, local noetherian domain, and its topology is its natural m-adic topology, where $\mathfrak{m}$ is the maximal ideal of $B$. In particular, $B$ is not 
a field since $B$ is not discrete. Therefore, as $B$ is open in the topological field $K$, the topology of $K$ is defined by a complete, discrete valuation [12, Theorem 6].

THEOREM 2. If $K$ is an indiscrete, Hausdorff topological division ring, then the topology of $K$ is given by a complete, discrete valuation and $K$ is finite-dimensional over its center $C$ if and only if $K$ is locally centrally linearly compact; in this case, $C$ is indiscrete, and hence its topology is given by a complete, discrete valuation.

Proof. Necessity. As $K$ is finite-dimensional over $C$, the valuation induced on $C$ by that of $K$ is not the improper valuation; hence as $C$ is closed, the topology of $C$ is given by a complete, discrete valuation $v$. Let $e_{1}, \cdots, e_{n}$ be a basis of $K$ over $C$ such that $e_{1}=1$, and let $e_{i} e_{j}=\sum_{k=1}^{n} \alpha_{i j k} e_{k}$. Let $\lambda \in C$ be such that $v(\lambda) \geqq 0$ and $v(\lambda) \geqq-$ $\min \left\{v\left(\alpha_{i j k}\right): 1 \leqq i, j, k \leqq n\right\}$. Let $f_{1}=1$ and $f_{k}=\lambda e_{k}$ for $2 \leqq k \leqq n$. Let $V$ be the valuation ring of $C$, and for each $m \geqq 0$ let $V_{m}=\{x \in$ $V: v(x) \geqq m\}$. Let $B=V f_{1}+\cdots+V f_{n}$, and for each $m \geqq 0$ let $\mathfrak{b}_{m}=$ $V_{m} f_{1}+\cdots+V_{m} f_{n}$. Easy calculations establish that $B$ is a ring and that $\mathfrak{b}_{m}$ is an ideal of $B$ for each $m \geqq 0$. By [2, Theorem 2, p. 18], $F:\left(\lambda_{1}, \cdots, \lambda_{n}\right) \mapsto \sum_{i=1}^{n} \lambda_{i} f_{i}$ is a topological isomorphism from the $C$ vector space $C^{n}$ onto the $C$-vector space $K$. Hence $B$ is an open subring of $K$, and $\left(\mathfrak{b}_{m}\right)_{m \geqq 0}$ is a fundamental system of neighborhoods of zero in $B$, each an ideal of $B$. We saw earlier that $V$ is strictly linearly compact; hence as $B=F\left(V^{n}\right), B$ is a strictly linearly compact $V$-module and, a fortiori, is a centrally linearly compact ring.

Sufficiency. By Lemma 1, there is an open, centrally linearly compact subring $B$ that contains 1 . Let $\mathfrak{x}$ be the radical of $B$. As the $\mathrm{r}$-adic topology is the given indiscrete topology of $B$ by Lemma 2, there exists a nonzero $a \in B$ such that $\lim a^{n}=0$. Let $K_{0}$ be the closed subfield generated by $C$ and $a$, let $B_{0}=K_{0} \cap B$, and let $\mathfrak{x}_{0}$ be the radical of $B_{0}$. Since the open left ideals of $B$ form a fundamental system of neighborhoods of zero for $B$, the open ideals of $B_{0}$ form a fundamental system of neighborhoods of zero for $B_{0}$. Moreover, the center $C_{B}$ of $B$ is simply $C \cap B$; indeed, if $c \in C_{B}$ and if $x \in K$, then $a^{n} x \in B$ for some $n$ as $\lim a^{n} x=0$, whence $\left(a^{n} x\right) c=c\left(a^{n} x\right)=\left(c a^{n}\right) x=$ $\left(a^{n} c\right) x$, so $x c=c x$. Thus $C_{B}=C \cap B \subseteq K_{0} \cap B=B_{0}$, so $B_{0}$ is a closed $C_{B}$-submodule of $B$ and hence is a strictly linearly compact $C_{B}$-module. Consequently, $B_{0}$ is a strictly linearly compact ring, so by Lemma 2, the topology of $B_{0}$ is the $\mathfrak{r}_{0}$-adic topology, and $\mathfrak{x}$ and $\mathfrak{r}_{0}$ are respectively the set of topological nilpotents in $B$ and $B_{0}$, whence $\mathfrak{r}_{0}=\mathfrak{x} \cap B_{0}$. Hence $\bigcap_{n=1}^{\infty}\left(\mathfrak{x}_{0}^{n} B\right)^{-} \subseteq \bigcap_{n=1}^{\infty} \mathfrak{x}^{n}=(0)$. As the topology of $B_{0}$ is indiscrete, $\mathfrak{x}_{0}^{2} \neq(0)$, so $\mathfrak{x}_{0}^{2} B$ is open as it contains a nonzero left ideal of $B$. By 
[13, Theorem 10], $\mathfrak{x}_{0} B$ is a finitely generated $B_{0}$-module; let $\mathfrak{x}_{0} B=$ $B_{0} x_{1}+\cdots+B_{0} x_{m}$. Also as $B$ is a strictly linearly compact $C_{B}$-module and as $\mathfrak{r}_{0} B$ is open, $B / \mathfrak{r}_{0} B$ is an artinian $C_{B}$-module, hence an artinian $B_{0}$-module; now $B / \mathfrak{x}_{0} B$ admits the structure of $B_{0} / \mathfrak{x}_{0}$-module, and $B_{0} / \mathfrak{x}_{0}$ is a field by Lemma 2; consequently $B / \mathfrak{r}_{0} B$ is an artinian, therefore, finite-dimensional, and hence noetherian $B_{0} / \mathfrak{x}_{0}$-vector space; thus $B / \mathfrak{x}_{0} B$ is a noetherian $B_{0}$-module. Let $x_{m+1}, \cdots, x_{n} \in B$ be such that $B=$ $B_{0} x_{m+1}+\cdots+B_{0} x_{n}+\mathfrak{r}_{0} B$. Then $B=B_{0} x_{1}+\cdots+B_{0} x_{n}$. Consequently, $x_{1}, \cdots, x_{n}$ is a set of generators of the $K_{0}$-vector space $K$, for if $z \in K$, there exists $t$ such that $a^{t} z \in B$, whence $a^{t} z=b_{1} x_{1}+\cdots+b_{n} x_{n}$ where $b_{i} \in B_{0}$, and thus $z=\left(a^{-t} b_{1}\right) x_{1}+\cdots+\left(a^{-t} b_{n}\right) x_{n} \in K_{0} x_{1}+\cdots+$ $K_{0} x_{n}$. By [1, Theorem 16], the centralizer $K_{0}^{\prime}$ of $K_{0}$ has degree $\leqq n$ over $C$. But $K_{0}^{\prime} \supseteqq K_{0}$ as $K_{0}$ is commutative. Moreover, the topology of $K_{0}$ is given by a discrete valuation by Theorem 1 , as $B_{0}$ is an open, strictly linearly compact subring. Therefore, as $\left[K_{0}: C\right] \leqq n$, the valuation induced on $C$ is not the improper valuation; hence the topology of $C$ is given by a complete, discrete valuation. As

$$
[K: C]=\left[K: K_{0}\right]\left[K_{0}: C\right] \leqq n^{2},
$$

the given topology of $K$ is the only topology for which $K$ is a Hausdorff topological vector space over $C$ [2, Theorem 2, p. 18]; by valuation theory, that topology is given by a complete, discrete valuation.

The idea of using [1, Theorem 16] is suggested by Kaplansky's treatment of locally compact division rings in [8].

Jacobson's theorem concerning totally disconnected locally compact division rings follows at once from Theorem 2. Indeed, if $K$ is an indiscrete, totally disconnected, locally compact division ring, then $K$ contains a compact open subring $B$ [9, Lemma 4]; the open ideals of $B$ form a fundamental system of neighborhoods of zero [7, Lemmas 9 and 10], and therefore the compact ring $B$ is clearly centrally linearly compact; by Theorem $2, K$ is finite-dimensional over its center, which is indiscrete, and the topology of $K$ is given by a complete, discrete valuation.

\section{REFERENCES}

1. E. Artin and G. Whaples, The theory of simple rings, Amer. J. Math., 65 (1943), 87-107.

2. N. Bourbaki, Espaces vectoriels topologiques, Ch. 1-2, 2nd ed., Hermann, Paris, 1966.

3. - Algèbre commutative, Ch. 3-4, Hermann, Paris, 1961.

4. —, Algèbre commutative, Ch. 5-6, Hermann, Paris, 1964.

5. N. Jacobson, Totally disconnected locally compact rings, Amer. J. Math., 58 (1936), 433-449.

6. Structure of Rings, Amer. Math. Soc. Colloq. Publ., vol. 37, Providence, R. I., 1956.

7. Irving Kaplansky, Topological rings, Amer. J. Math., 69 (1947), 153-183. 
8. Irving Kaplansky, Topological methods in valuation theory, Duke Math. J., 14 (1947), $527-541$.

9. _ـ Locally compact rings, Amer. J. Math., 70 (1948), 447-459.

10. Horst Leptin, Linear kompakte Moduln und Ringe, Math. Z., 62 (1955), 241-267.

11. Seth Warner, Compact rings, Math. Ann., 145 (1962), 52-63.

12. — Openly embedding local noetherian domains, J. Reine Angew. Math., 253 (1972), 146-151.

13. L Linearly compact rings and modules, Math. Ann., 197 (1972), 29-43.

Received June 12, 1972.

DUKE UNIVERSITY 


\section{PACIFIC JOURNAL OF MATHEMATICS}

\section{EDITORS}

RICHARD ARENS (Managing Editor)

University of California

Los Angeles, California 90024

R. A. Beaumont

University of Washington

Seattle, Washington 98105
J. DUGUNDJI*

Department of Mathematics

University of Southern California

Los Angeles, California 90007

D. Gilbarg and J. Milgram

Stanford University

Stanford, California 94305

\section{ASSOCIATE EDITORS}
E. F. BECKENBACH
B. H. NeumanN
F. WOLF
K. YosHIDA

\section{SUPPORTING INSTITUTIONS}

UNIVERSITY OF BRITISH COLUMBIA
CALIFORNIA INSTITUTE OF TECHNOLOGY
UNIVERSITY OF CALIFORNIA
MONTANA STATE UNIVERSITY
UNIVERSITY OF NEVADA
NEW MEXICO STATE UNIVERSITY
OREGON STATE UNIVERSITY
UNIVERSITY OF OREGON
OSAKA UNIVERSITY

UNIVERSITY OF BRITISH COLUMBIA

UNIVERSITY OF CALIFORNIA

MONTANA STATE UNIVERSITY

UNIVERSITY OF NEVADA

OREGON STATE UNIVERSITY

OSAKA UNIVERSITY
UNIVERSITY OF SOUTHERN CALIFORNIA

STANFORD UNIVERSITY

UNIVERSITY OF TOKYO

UNIVERSITY OF UTAH

WASHINGTON STATE UNIVERSITY

UNIVERSITY OF WASHINGTON

\section{AMERICAN MATHEMATICAL SOCIETY} NAVAL WEAPONS CENTER

* C. R. DePrima California Institute of Technology, Pasadena, CA 91109, will replace J. Dugundji until August 1974. 


\section{Pacific Journal of Mathematics}

\section{Vol. 48, No. $1 \quad$ March, 1973}

Jan Aarts and David John Lutzer, Pseudo-completeness and the product of Baire

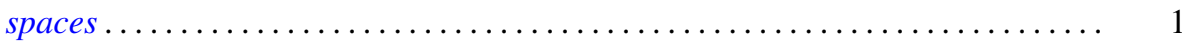

Gordon Owen Berg, Metric characterizations of Euclidean spaces ............ 11

Ajit Kaur Chilana, The space of bounded sequences with the mixed topology ..... . 29

Philip Throop Church and James Timourian, Differentiable open maps of

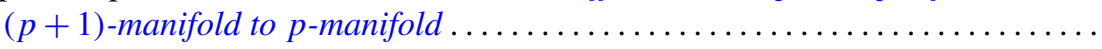

P. D. T. A. Elliott, On additive functions whose limiting distributions possess a finite

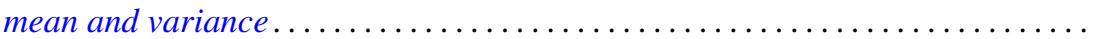

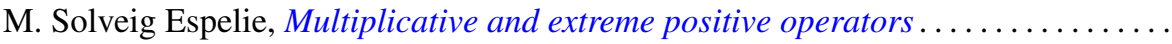

Jacques A. Ferland, Domains of negativity and application to generalized convexity

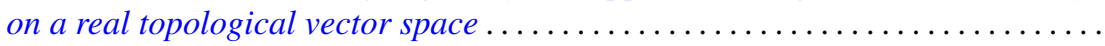

Michael Benton Freeman and Reese Harvey, A compact set that is locally holomorphically convex but not holomorphically convex ...............

Roe William Goodman, Positive-definite distributions and intertwining

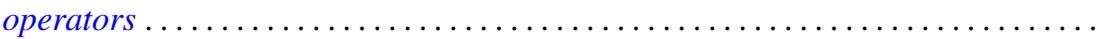

Elliot Charles Gootman, The type of some $C^{*}$ and $W^{*}$-algebras associated with

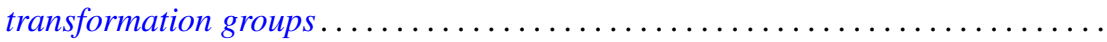

David Charles Haddad, Angular limits of locally finitely valent holomorphic

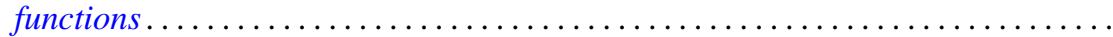

William Buhmann Johnson, On quasi-complements .

William M. Kantor, On 2-transitive collineation groups of finite projective spaces...

Joachim Lambek and Gerhard O. Michler, Completions and classical localizations of right Noetherian rings

Kenneth Lamar Lange, Borel sets of probability measures ......

David Lowell Lovelady, Product integrals for an ordinary differential equation in a Banach space

Jorge Martinez, A hom-functor for lattice-ordered groups .........

W. K. Mason, Weakly almost periodic homeomorphisms of the two sphere ....

Anthony G. Mucci, Limits for martingale-like sequences .......

Eugene Michael Norris, Relationally induced semigroups ...

Arthur E. Olson, A comparison of c-density and $k$-density ......

Donald Steven Passman, On the semisimplicity of group rings of linear groups.

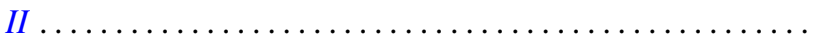

Charles Radin, Ergodicity in von Neumann algebras .

P. Rosenthal, On the singularities of the function generated by the Bergman operator of the second kind.

Arthur Argyle Sagle and J. R. Schumi, Multiplications on homogeneous spaces,

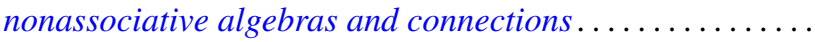

Leo Sario and Cecilia Wang, Existence of Dirichlet finite biharmonic functions on

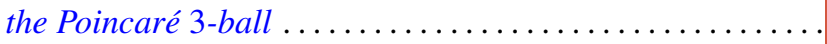

Ramachandran Subramanian, On a generalization of martingales due to Blake ..

Bui An Ton, On strongly nonlinear elliptic variational inequalities.

Seth Warner, A topological characterization of complete, discretely valued

fields. 